\title{
A New Fundamental and Numerical Method for the Fractional Partial Differential Equations
}

\author{
Tie Liu \\ Department of Mathematics and Statistics, Ankang University, Ankang, Shaanxi, \\ China, 725000 \\ Liutie@aku.edu.cn
}

\begin{abstract}
Fractional order has the characteristics of memory and non-locality and it is different with integer order. Therefore, fractional differential equations can be used to describe some abnormal natural phenomena. At the same time, how to solve the fractional order partial differential equation and differential equations with fractional order has become a very important research field. Besides analytic solution, it is also important to investigate the numerical methods for fractional differential equations. In the paper, fundamental solution of the time fractional partial differential equation has been deduced, which is derived by Furrier transform and Laplace transform. According to the simulation, there is little difference between numerical solution and the exact solution when the solution is the time variable function. The results show the validity of the method.
\end{abstract}

Keywords: fractional partial differential function, fundamental solution, numerical solution, comparison

\section{Introduction}

With the development of research on natural science and economics, deficiencies of integer order differential equations [1] has been revealed in the simulation of concrete problems. Then, it is needed to explore and study the mathematical tool which has more general significance. Among them, fractional calculus [2-4] is one of the mathematical problems worthy of further study. Fractional derivative [5-6] with real number as the order is defined by the integral method, and it is the characteristics of memory and nonlocality [7-8], which is different with integer order. Therefore, compared with the integer order differential equation, fractional differential equations [9-10] can meet the special needs in some conditions.

Fractional partial differential equation can be used to describe some abnormal natural phenomena [11-13]. Fractional diffusion equation can be used to describe the abnormal slow diffusion phenomenon [14-16] in porous media with fractal structures, and fractional advection diffusion equation can be used to describe the anomalous osmotic phenomenon of fluid in medium [17-18]. Therefore, research on fractional partial differential equation application attracts more and more extensive attention, especially on the study of fractional diffusion equation [19-20] and fractional advection diffusion equation [21-22].

With the wide application of fractional calculus system, how to solve the fractional order partial differential equation and differential equations with fractional order has become a very important research field. Laplace transform and Mellin transform can be used to find the fundamental solution of the time fractional advection-diffusion equation in the space of half plane [23]. By using Furrier transform and Laplace transform, fundamental solution of time fractional diffusion equation of the whole plane can be deduced. The fundamental solution is obtained through the Laplace inverse transform of Mittag-Leffler function. 
However, it is similar as integer order differential equation, and fractional solutions of only a few fractional order differential equations can be found by analytical means. So, it is also important to investigate the numerical methods for fractional differential equations. At the same time, due to the special characteristic of fractional differential equations, calculation of fractional differential equations will need great workload, and how to get the effective numerical methods in solving fractional differential equations is an important problem.

The main work of the paper is to deduce the fundamental solution of the time fractional partial differential equation [24-25]. It includes some conditions: the time fractional diffusion equation, time fractional reaction-diffusion equations, the time fractional advection-diffusion equation and their corresponding integer order partial differential equation. The fundamental solution is derived by Furrier transform and Laplace transform. The main contribution of the paper is to get a fundamental solution. In the paper, we also use a numerical method to calculate the solution of the function. The remainder of the paper is shown as the following: the function to be solved is shown in Section 2. Preliminary knowledge is described in Section 3. Fundamental solution is expressed in Section 4. The experiment is shown in Section 5 and the conclusion is described in Section 6.

\section{The Function}

The function we considered can be shown as the following:

$$
\frac{\partial^{\alpha} u(x, t)}{\partial t^{\alpha}}=-\lambda^{2} u(x, t)-v \frac{\partial u(x, t)}{\partial x}+D \frac{\partial^{2} u(x, t)}{\partial x^{2}}
$$

$0<\alpha \leq 1$

$u(x, 0)=f(x)$

$u( \pm \infty, t)=0$

$$
\begin{aligned}
& -\infty<x \leq \infty \\
& t>0
\end{aligned}
$$

Where, $\lambda \geq 0, v \geq 0, D>0, \frac{\partial^{\alpha} u(x, t)}{\partial t^{\alpha}}$ is the Caputo Fractional Partial Differential Equations, and the definition can be describes as follows:

$$
\frac{\partial^{\alpha} f(t)}{\partial t^{\alpha}}= \begin{cases}\frac{d^{n} f(t)}{d t^{n}} & \alpha=n \in Z \\ \frac{1}{\Gamma(n-\alpha)} \int_{0}^{t}(t-\tau)^{n-\alpha-1} \frac{d^{n} f(\tau)}{d \tau^{n}} d \tau, & n-1<\alpha<n\end{cases}
$$

When $v \neq 0$, the equation can be transformed as the following to get the results easily:

$$
u(x, t)=C(\xi, t) e^{v \xi /(2 \sqrt{D})}, \quad \xi=\frac{x}{\sqrt{D}}
$$

Then the equation (1) can be described as follows:

$$
\frac{\partial^{\alpha} C(\xi, t)}{\partial t^{\alpha}}=-m^{2} C(\xi, t)+\frac{\partial^{2} C(\xi, t)}{\partial \xi^{2}}
$$

Where, $m^{2}=\frac{v^{2}}{4 D}+\lambda^{2}$.

Here, we just take condition of $v=0$ into consideration, that is to say that the function is expressed as the following: 


$$
\frac{\partial^{\alpha} C(x, t)}{\partial t^{\alpha}}=-\lambda^{2} C(x, t)+D \frac{\partial^{2} C(x, t)}{\partial x^{2}}, \quad x \in \Omega, t>0
$$

\section{Preliminary Knowledge}

(1) Laplace transform definition

$$
F(s)=L\{f(t) ; s\}=\int_{0}^{\infty} f(t) d t
$$

And its inverse transformation is the following:

$$
F(t)=L^{-1}\{f(s) ; t\}=\frac{1}{2 \pi i} \int_{c-i \infty}^{c+i \infty} e^{s t} f(s) d t \quad c=\operatorname{Re}(s)
$$

(2) Fourier transform definition

$$
H(w)=F\{h(t) ; w\}=\int_{-\infty}^{\infty} e^{i w t} h(t) d t
$$

And its inverse transform is the following:

$$
h(t)=F^{-1}\{H(w) ; t\}=\frac{1}{2 \pi} \int_{-\infty}^{\infty} e^{-i w t} H(w) d w
$$

(3) Definition of Caputo fractional derivative

$$
{ }_{0}^{c} D_{t}^{\alpha} f(t)=\left\{\begin{array}{lr}
\frac{1}{\Gamma(n-\alpha)} \int_{0}^{t} \frac{f^{(n)}(\tau)}{(t-\tau)^{\alpha-n+1}} d(\tau) & n-1<\alpha<n \\
\frac{d^{(n)} f(t)}{d t^{n}} & \alpha=n
\end{array}\right.
$$

And the Laplace transform is as the following:

$$
L\left\{{ }_{0}^{c} D_{t}^{\alpha} f(t) ; s\right\}=s^{\alpha} F(s)-\sum_{k=0}^{n-1} s^{\alpha-k-1} f^{(k)}(0) \quad n-1<\alpha<n
$$

(4) Wright function

$$
W(-z ; \alpha, \beta)=\sum_{k=0}^{\infty} \frac{z^{k}}{\Gamma(\alpha k+\beta) k !} .
$$

There is also a definition as follows:

$$
M_{\alpha}(Z)=\sum_{k=0}^{\infty} \frac{(-1)^{k} z^{k}}{\Gamma(-\alpha k+(1-\alpha)) k !}
$$

It is defined as $M_{\alpha}(z)$, and then, if $\alpha=1, M_{1}(z)=\delta(z-1)$. Where, $\delta(z)$ is Dirac $\delta$ function.

\section{The basic Solution of the Time Fractional Partial Differential Equations}

Some definition:

$$
u(x, t)=C(\zeta, t) \exp \left(\frac{v \zeta}{2 \sqrt{D}}\right)
$$




$$
\lambda^{2}=\left(v^{2} / 4 D\right)+m^{2}
$$

If we give the formula as follows:

$$
E_{\alpha}\left(c t^{\alpha}\right) \leftarrow \stackrel{L}{\longrightarrow} \frac{s^{\alpha-1}}{s^{\alpha}-c}, \quad \operatorname{Re}(s)>|c|^{\frac{1}{\alpha}}
$$

Where, $c \in C$, and $E_{\alpha}$ is a function.

$$
E_{\alpha}=\sum_{n=0}^{\infty} \frac{z^{n}}{\Gamma(\alpha n+1)} \quad \alpha>0, z \in C
$$

Then, we can get:

$$
\hat{u}(k, t)=E_{\alpha}\left[-\left(D k^{2}+\lambda^{2}\right) t^{\alpha}\right] \hat{f}(k)
$$

With the inverse Fourier transform,

$$
\begin{aligned}
u(x, t) & =\frac{1}{2 \pi} \int_{-\infty}^{+\infty} e^{-i k x} E_{\alpha}\left[-\left(D k^{2}+\lambda^{2}\right) t^{\alpha}\right] \hat{f}(k) d k \\
& =\int_{-\infty}^{+\infty} G_{C}^{(\alpha)}(x-y, t) f(y) d y
\end{aligned}
$$

Where,

$$
G_{C}^{(\alpha)}(x, t)=\frac{1}{2 \pi} \int_{-\infty}^{+\infty} e^{-i k x} E_{\alpha}\left[-\left(D k^{2}+\lambda^{2}\right) t^{\alpha}\right] d k
$$

This is called the Green function or the basic solution. It should meet the demand of the following condition:

$$
G_{C}^{(\alpha)}(k, t)=E_{\alpha}\left[-\left(D k^{2}+\lambda^{2}\right) t^{\alpha}\right]
$$

And

$$
\bar{G}(k, s)=\frac{s^{\alpha-1}}{s^{\alpha}+\left(D k^{2}+\lambda^{2}\right)}
$$

This equation can be transformed to the following form:

$$
\bar{G}(k, s)=E\left[-\left(D k^{2}+\lambda^{2}\right) t\right]
$$

Consider the Fourier and Laplace relationships:

$$
\frac{1}{2 \sqrt{\pi}} u^{-\frac{1}{2}} e^{-\frac{x^{2}}{4 u}} \longleftarrow F
$$

And

$$
t^{-\alpha} M_{\alpha}\left(\frac{u}{t^{\alpha}}\right) \stackrel{L}{\longleftarrow} \rightarrow s^{\alpha-1} e^{-u s^{\alpha}}
$$

Then the Green Function can be described as:

$$
G_{C}(x, t)=\frac{1}{2 \sqrt{\pi}}(D t)^{-\frac{1}{2}} e^{\frac{-x^{2}}{4 D t}} e^{-\lambda^{2} t}
$$




$$
G(x, t)=\frac{t^{-\alpha}}{2 \sqrt{\pi}} \int_{0}^{\infty} e^{\frac{-x^{2}}{4 u}} e^{-\lambda^{2} u} u^{-\frac{1}{2}} M_{\alpha}\left(\frac{u}{t^{\alpha}}\right) d u
$$

If set $u=\kappa t^{\alpha}$, then

$$
\begin{aligned}
G(x, t) & =\frac{1}{2 \sqrt{\pi t^{\alpha}}} \int_{0}^{\infty} e^{\frac{-x^{2}}{4 t^{\alpha} \kappa}-\lambda^{2} t^{\alpha} \kappa} \kappa^{-\frac{1}{2}} M_{\alpha}(\kappa) d \kappa \\
& =\frac{1}{2 \sqrt{\pi t}} \int_{0}^{\infty} e^{\frac{-x^{2}}{4 t^{\alpha} \kappa}-\left(\frac{\nu^{2}}{4 D}+\lambda^{2}\right) t^{\alpha} \kappa} \kappa^{-\frac{1}{2}} M_{\alpha}(\kappa) d \kappa
\end{aligned}
$$

If $\lambda=0$, it will be the time fractional advection-dispersion function. Then $G(x, t)$ can be described as:

$$
G(x, t)=\frac{1}{2 \sqrt{\pi t}} \int_{0}^{\infty} e^{\frac{-x^{2}}{4 t^{\alpha} \kappa}-\frac{\nu^{2}}{4 D} t^{\alpha} \kappa} \kappa^{-\frac{1}{2}} M_{\alpha}(\kappa) d \kappa
$$

If $\alpha=1$, the function will be the integer partial differential equation, and the $G(x, t)$ can be described as:

$$
\begin{aligned}
G(x, t) & =\frac{1}{2 \sqrt{\pi t}} \int_{0}^{\infty} e^{\frac{-x^{2}}{4 t \kappa}-\left(\frac{v^{2}}{4 D}+\lambda^{2}\right) t \kappa} \kappa^{-\frac{1}{2}} M_{1}(\kappa) d \kappa \\
& =\frac{1}{2 \sqrt{\pi t}} \int_{0}^{\infty} e^{\frac{-x^{2}}{4 t \kappa}-\left(\frac{v^{2}}{4 D}+\lambda^{2}\right) t \kappa} \kappa^{-\frac{1}{2}} \delta(\kappa-1) d \kappa \\
& =\frac{1}{2 \sqrt{\pi t}} e^{\frac{-x^{2}}{4 t}-\left(\frac{v^{2}}{4 D}+\lambda^{2}\right) t}
\end{aligned}
$$

When $\lambda=0$, the $G(x, t)$ can be described as:

$$
\begin{aligned}
G(x, t) & =\frac{1}{2 \sqrt{\pi t}} \int_{0}^{\infty} e^{\frac{-x^{2}}{4 t \kappa}-\left(\frac{v^{2}}{4 D}+\lambda^{2}\right) t \kappa} \kappa^{-\frac{1}{2}} M_{1}(\kappa) d \kappa \\
& =\frac{1}{2 \sqrt{\pi t}} \int_{0}^{\infty} e^{\frac{-x^{2}}{4 t \kappa}-\left(\frac{v^{2}}{4 D}+\lambda^{2}\right) t \kappa} \kappa^{-\frac{1}{2}} \delta(\kappa-1) d \kappa \\
& =\frac{1}{2 \sqrt{\pi t}} e^{\frac{-x^{2}}{4 t}-\frac{v^{2} t}{4 D}}
\end{aligned}
$$

When $v=0, D>0$, the $G(x, t)$ can be described as:

$$
G(x, t)=\frac{1}{2 \sqrt{\pi t}} \int_{0}^{\infty} e^{\frac{-x^{2}}{4 t^{\alpha} \kappa}-\lambda^{2} t^{\alpha} \kappa} \kappa^{-\frac{1}{2}} M_{\alpha}(\kappa) d \kappa
$$


If $\alpha=1$, the $G(x, t)$ can be described as:

$$
G(x, t)=\frac{1}{2 \sqrt{\pi t}} e^{\frac{-x^{2}}{4 t}-\lambda^{2} t}
$$

When $\lambda=0, v=0, D>0$, the $G(x, t)$ can be described as:

$$
G(x, t)=\frac{1}{2 \sqrt{\pi t^{\alpha}}} M_{\frac{\alpha}{2}}(\kappa)\left(\frac{|x|}{\sqrt{D t^{\alpha}}}\right)
$$

Then if $\alpha=1$, the $G(x, t)$ can be described as:

$$
G(x, t)=\frac{1}{2 \sqrt{\pi}} e^{\frac{-x^{2}}{4 D t}}
$$

\section{Comparison of the Results}

Equation (36) and equation (37) are solved by the method proposed in the paper. Besides, the numerical method [26-27] is also used to solve the both of the two equations. In addition, the boundary value condition is transferred into homogeneous condition, and the first 15 orders are adopted. The solution of the two methods is compared in the figure 1 to figure 10. In all the Figures, the line is the exact solution and the "*" represents the numerical solution.

Equation 1:

$$
\begin{cases}\frac{\partial^{\alpha} u(x, t)}{\partial t^{\alpha}}+\lambda^{2} u(x, t)-D \frac{\partial^{2} u(x, t)}{\partial x^{2}}=0 & x \in(0, L), t>0 \\ u(x, 0)=f(x), & x \in(0, L), \\ u(0, t)=u(L, t)=0, & t>0\end{cases}
$$

Equation 2:

$$
\left\{\begin{array}{cl}
\frac{\partial^{\alpha} u(x, t)}{\partial t^{\alpha}}+\lambda^{2} u(x, t)-D \frac{\partial^{2} u(x, t)}{\partial x^{2}}=0 & x \in(0, L), t>0 \\
u(x, 0)=f(x), & x \in(0, L), \\
u_{x}(0, t)=u_{x}(L, t)=0, & t>0
\end{array}\right.
$$

Figure 1 to Figure 4 show the comparison of the numerical solution and the exact solution of equation 1 when the solution is the time variable function. It can be seen from the figures that there is little difference between the numerical solution and the exact solution. Figure 5 to Figure 8 show the comparison of the numerical solution and the exact solution of equation 2 . The numerical results also have little difference to the exact solution. 


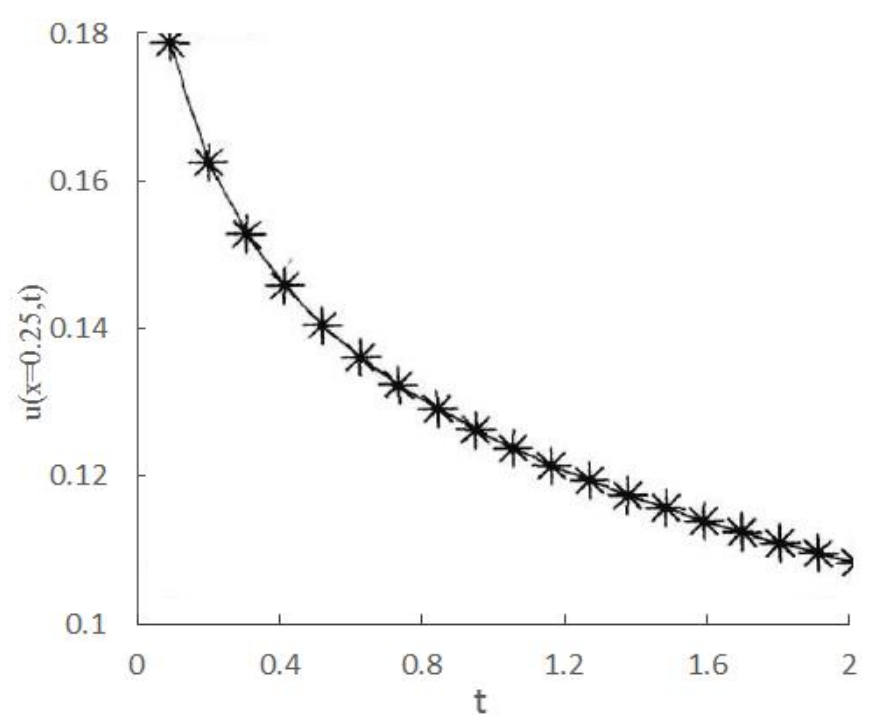

Figure 1. $\lambda=1 / 8, x=0.25, \alpha=0.25$

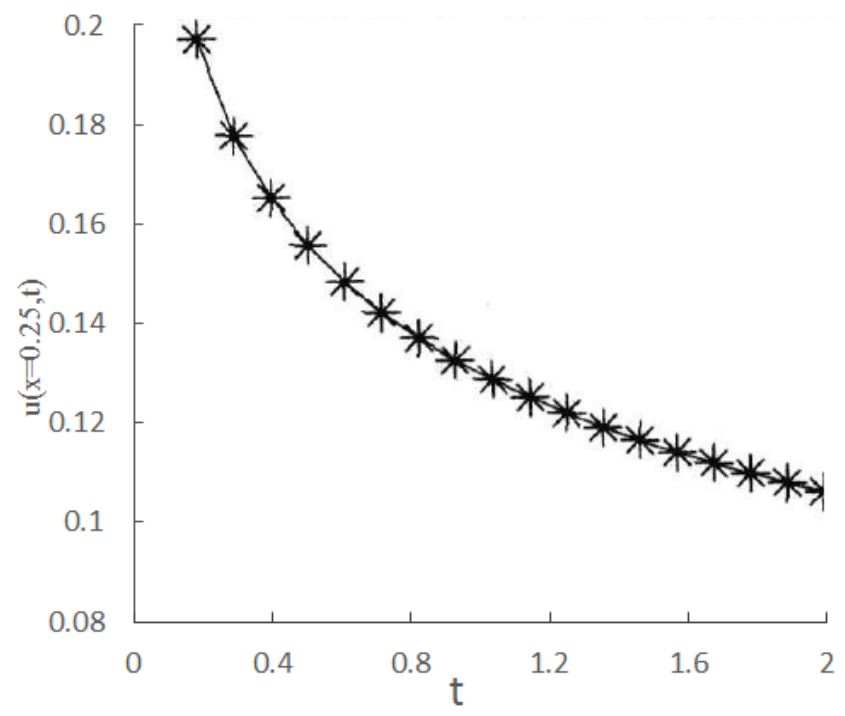

Figure 2. $\lambda=1 / 8, x=0.25, \alpha=0.5$

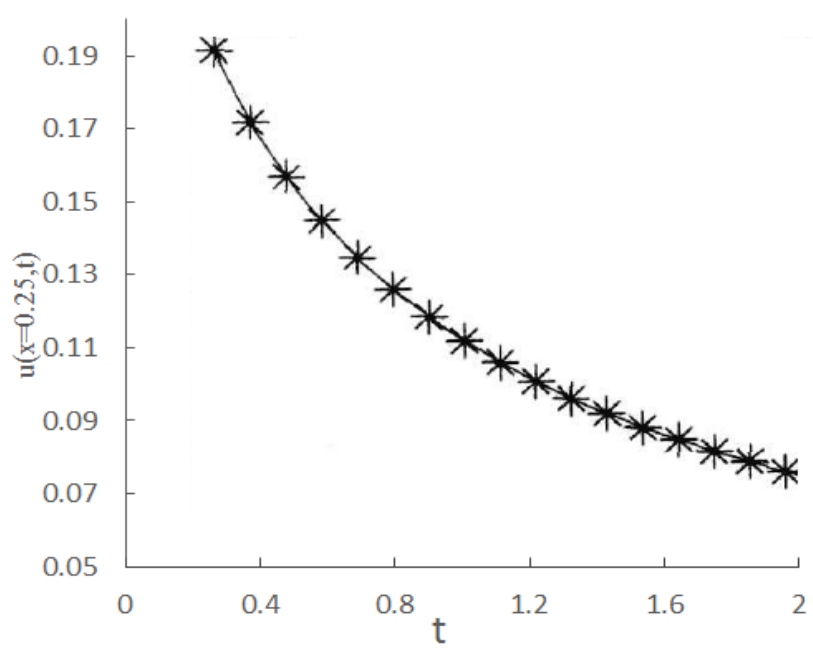

Figure 3. $\lambda=1 / 8, x=0.25, \alpha=0.75$ 


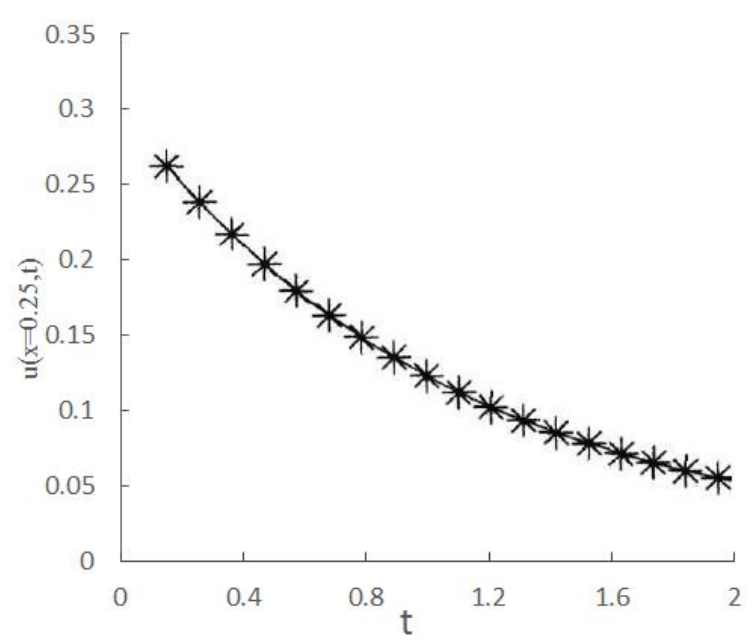

Figure 4. $\lambda=1 / 8, x=0.25, \alpha=1$

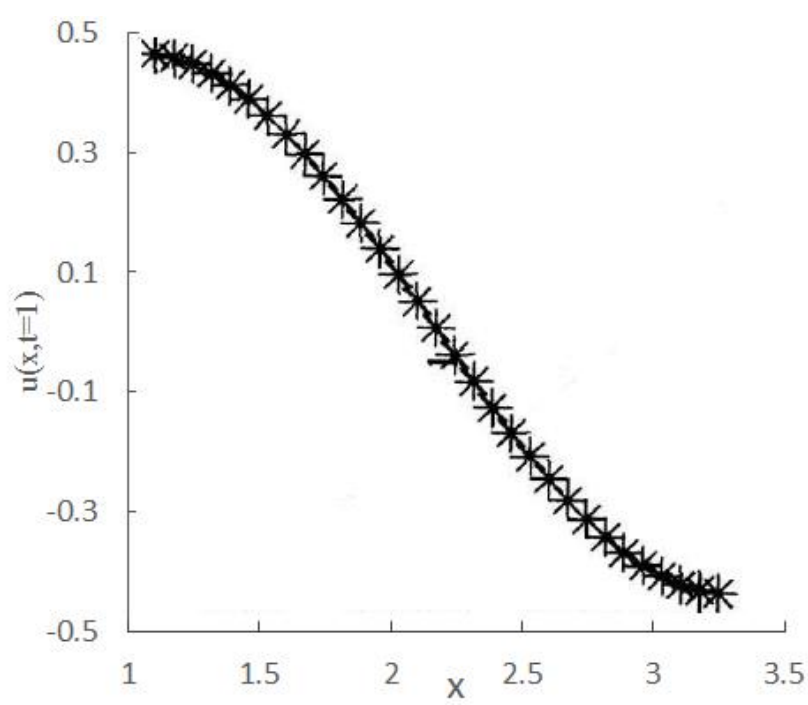

Figure 5. $\lambda=1 / 8, \alpha=0.25, t=1$

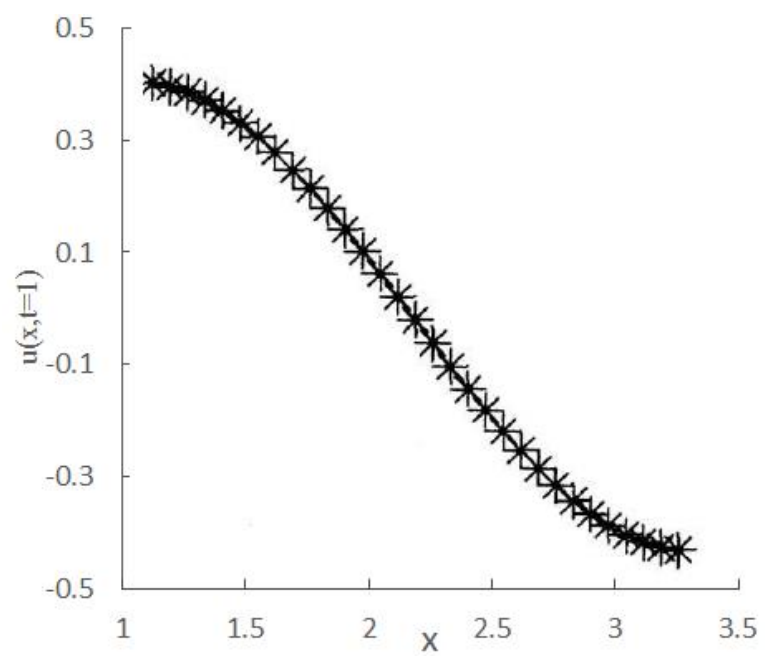

Figure. $6 \lambda=1 / 8, \alpha=0.5, t=1$ 


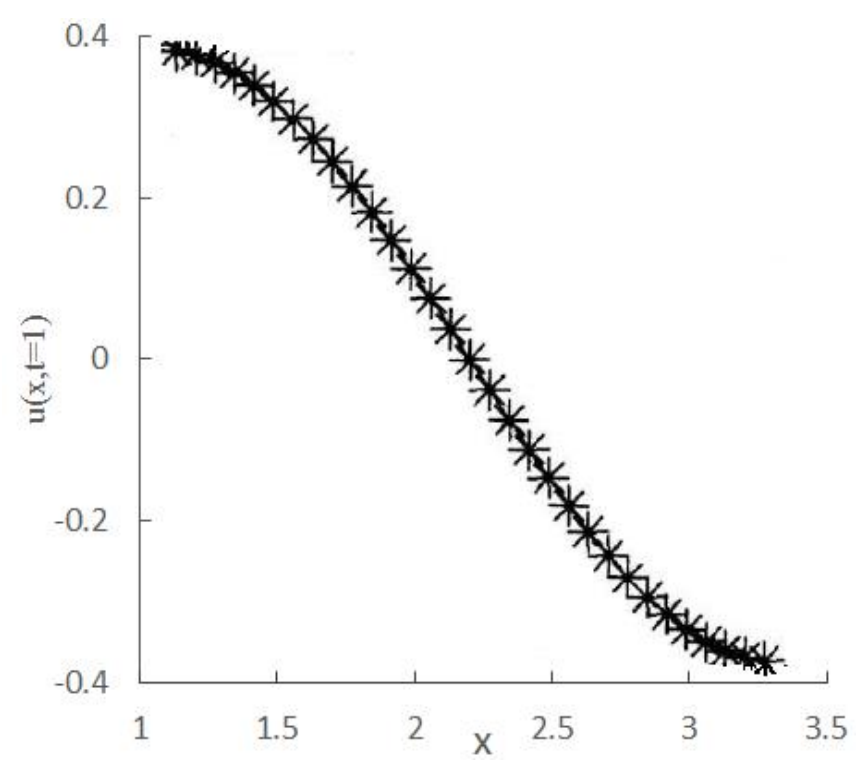

Figure 7. $\lambda=1 / 8, \alpha=0.75, t=1$

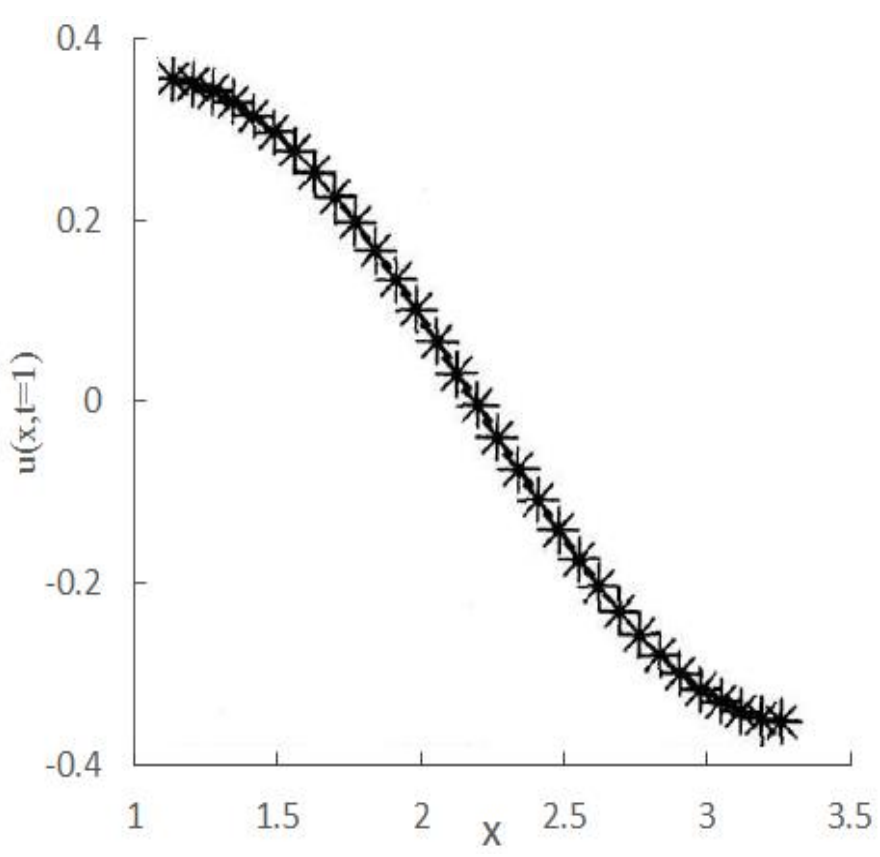

Figure 8. $\lambda=1 / 8, \alpha=1, t=1$

Figure 9 and 10 shows the comparison of numerical solution and the exact solution of equation 1 when the solution is the time variable function. It can be seen from the figures that there is little difference between numerical solution and the exact solution. When $t$ is small, value of solution increases with the increasing of $\alpha$, and when $t$ is big enough, value of solution decreases with the increasing of $\alpha$. 


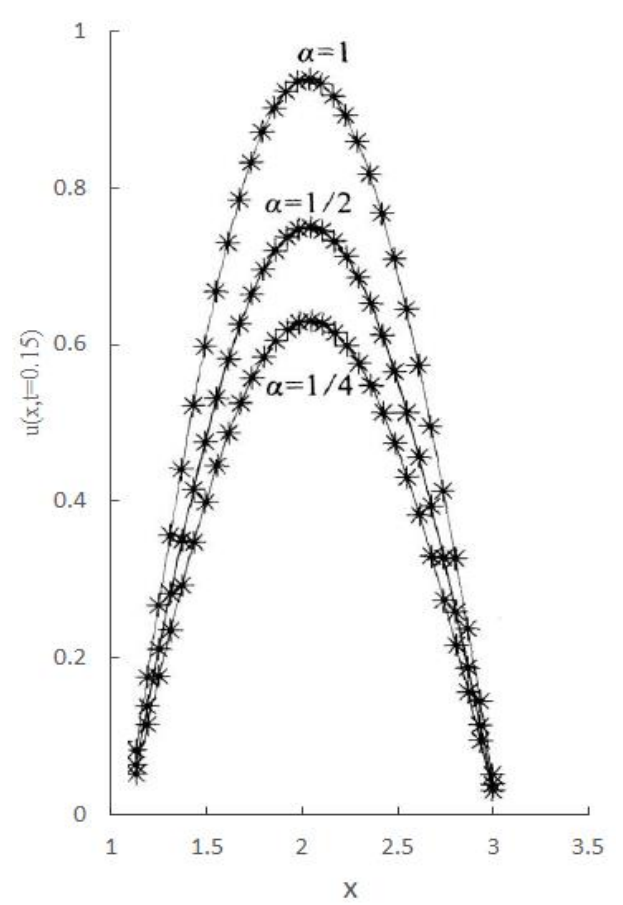

Figure 9. $\lambda=1 / 8, t=0.15$

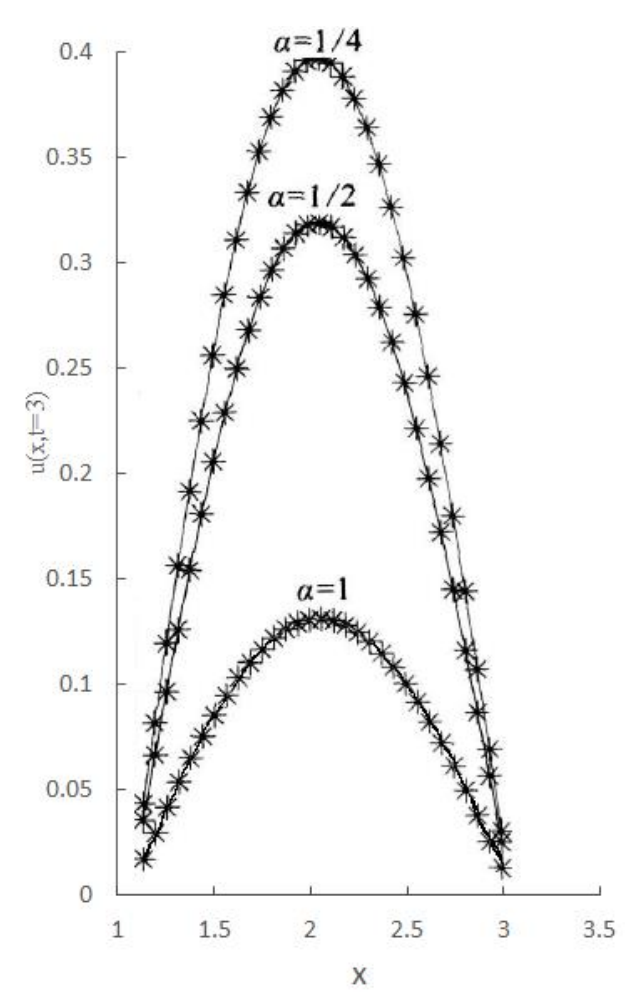

Figure 10. $\lambda=1 / 8, t=3$

\section{Conclusion}

Development of natural science and economics, deficiencies of integer order differential equations has been found. Fractional calculus has more general significance in describing some special problems, and it is one of the mathematical problems worthy of further study. Fractional order has the characteristics of memory and non-locality and it is 
different with integer order. Therefore, fractional differential equations can meet the special needs in some conditions, and it can be used to describe some abnormal natural phenomena. At the same time, how to solve the fractional order partial differential equation and differential equations with fractional order have become a very important research field. It is also important to investigate the numerical methods for fractional differential equations. How to get effective numerical methods in solving fractional differential equations is an important problem.

In the paper, fundamental solution of the time fractional partial differential equation has been deduced, which is derived by Furrier transform and Laplace transform. According to the simulation verification, validity of the method has been proved. There is little difference between numerical solution and the exact solution when the solution is the time variable function. Numerical solution and the exact solution decrease with the attenuation of time $t$. When $t$ is small, value of solution is increases with the increasing of $\alpha$, and when $t$ is big enough, value of solution decreases with the increasing of $\alpha$.

\section{References}

[1] S. Tomas, P. Igor, P. Ivo and B. Dagmar, "Data fitting using solutions of differential equations: Fractional-order model versus integer-order model", Proceedings of the 2012 13th International Carpathian Control Conference, ICCC 2012, (2012), pp. 703-710.

[2] Y. Ai-Min, Z. Ling, M. Xiao-Jun, Z. Yi-Jun and J. Ying, "The fractional calculus numerical algorithms and its application to the viscoelastic material problem", Journal of Chemical and Pharmaceutical Research, vol. 5, no. 12, (2013), pp. 821-828.

[3] T. Takahiro and O. Hiromitsu, "State-feedback control system using adaptive observer for continuoustime linear fractional calculus system", Proceedings of the SICE Annual Conference, (2013), pp. 18591864.

[4] G. Cottone, "A fractional calculus framework for the statistics of non-linear stochastic dynamic equations under stationary non-white excitation”, Safety, Reliability, Risk and Life-Cycle Performance of Structures and Infrastructures - Proceedings of the 11th International Conference on Structural Safety and Reliability, ICOSSAR 2013, (2013), pp. 1091-1098.

[5] F. Kwok Sau, "Fractional Langevin equation and Riemann-Liouville fractional derivative", European Physical Journal E, vol. 24, no. 2, (2007), pp. 139-143.

[6] Y. Meisam and H. Mohammad, "Application of fractional derivative in control functions", Proceedings of the INDICON 2008 IEEE Conference and Exhibition on Control, Communications and Automation, vol. 1, (2008), pp. 252-257.

[7] A. C. Galucio, J.-F. Deüi and R. Ohayon, "Finite element formulation of viscoelastic sandwich beams using fractional derivative operators", Computational Mechanics, vol. 33, no. 4, (2004), pp. 282-291.

[8] F. Luchko Yury, R. Margarita, J. J. Trujillo and M. Pilar Velasco, "Fractional models, non-locality, and complex systems", Computers and Mathematics with Applications, vol. 59, no. 3, (2010), pp. 1048-1056.

[9] S. Yu Lukashchuk, "An approximate solution method for ordinary fractional differential equations with the Riemann-Liouville fractional derivatives", Communications in Nonlinear Science and Numerical Simulation, vol. 19, no. 2, (2014), pp. 390-400.

[10] Z. Xingqiu, "Existence and iteration of positive solutions for high-order fractional differential equations with integral conditions on a half-line", Journal of Applied Mathematics and Computing, vol. 45, no. 1-2, (2014), pp. 137-150.

[11] S. Min, W. Zaihua and D. Maolin, "A modified multi-step differential transform method for solving fractional dynamic systems", Journal of Computational and Nonlinear Dynamics, vol. 8, no. 1, (2013).

[12] K. Justin and C. S. Drapaca, "A fractional pressure-volume model of cerebrospinal fluid dynamics in hydrocephalus", Conference Proceedings of the Society for Experimental Mechanics Series, vol. 4, (2014), pp. 179-184.

[13] J. Kauffman and S. Drapaca Corina, "A multiscale pressure-volume model of cerebrospinal fluid dynamics: Application to hydrocephalus", ASME 2013 2nd Global Congress on NanoEngineering for Medicine and Biology, NEMB 2013, (2013).

[14] L. S. Lucena, L. R. Da Silva, A. A. Tateishi, M. K. Lenzi, H. V. Ribeiro and E. K. Lenzi, "Solutions for a fractional diffusion equation with noninteger dimensions", Nonlinear Analysis: Real World Applications, vol. 13, no. 4, (2012), pp. 1955-1960.

[15] Y. Fan, F. Chu-Li and L. Xiao-Xiao, "A mollification regularization method for unknown source in time-fractional diffusion equation”, International Journal of Computer Mathematics, (2014).

[16] K. Mokhtar, A. Malik Salman and A. Al-Gwaiz Mohammed, "An inverse source problem for a two dimensional time fractional diffusion equation with nonlocal boundary conditions", Mathematical Methods in the Applied Sciences, vol. 36, no. 9, (2013), pp. 1056-1069. 
[17] P. A. Santoro, J. L. De Paula, E. K. Lenzi and L. R. Evangelista, “Anomalous diffusion governed by a fractional diffusion equation and the electrical response of an electrolytic cell”, Journal of Chemical Physics, vol. 135, no. 11, (2011).

[18] C. Jin, N. Junichi, Y. Masahiro and Y. Tomohiro, "Uniqueness in an inverse problem for a onedimensional fractional diffusion equation", Inverse Problems, vol. 25, no. 11, (2009).

[19] M. Mophou Gisèle, "Optimal control of fractional diffusion equation. Computers and Mathematics with Applications”, vol. 61, no. 1, (2011), pp. 68-78.

[20] D. René, M. Mophou Gisle and S. Valmorin Vincent, "Optimal control of a nonhomogeneous Dirichlet boundary fractional diffusion equation", Computers and Mathematics with Applications, vol. 62, no. 3, (2011), pp. 1472-1481.

[21] S. Shen, F. Liu, V. Anh, I. Turner and J. Chen, "A characteristic difference method for the variableorder fractional advection-diffusion equation”, Journal of Applied Mathematics and Computing, vol. 42, no. 1-2, (2013), pp. 371-386.

[22] Z. Yunying, D. Weihua and W. Yujiang, "A note on the finite element method for the space-fractional advection diffusion equation", Computers and Mathematics with Applications, vol. 59, no. 5, (2010), pp. 1718-1726.

[23] Y. Z. Povstenko, "Fundamental solutions to time-fractional advection diffusion equation in a case of two space variables", Mathematical Problems in Engineering, (2014).

[24] Z. Haixiang and H. Xuli, "Quasi-wavelet method for time-dependent fractional partial differential equation”, International Journal of Computer Mathematics, vol. 90, no. 11, (2013), pp. 2491-2507.

[25] A. Abdon and B. Samir Brahim, "Solving partial differential equation with space- and time-fractional derivatives via homotopy decomposition method", Mathematical Problems in Engineering, (2013).

[26] O. P. Agrawal, "Solution for a fractional diffusion-wave equation defined in a bounded domain", Nonlinear Dynamics, vol. 29, no. 1-4, (2002), pp. 145-155.

[27] R. Gorenflo and F. Mainardi, "Fractional calculus: integral and differential equations of fractional Order", Carpinteri A, Mainardi F. Fractals and Fractional Calculus in Continuum Mechanics. New York: Springer, (1997), pp. 223-276.

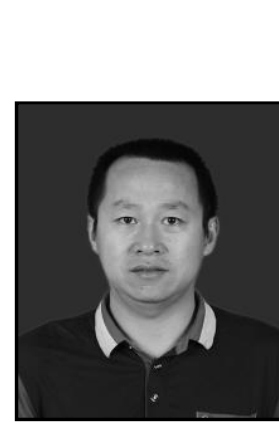

\section{Author}

Tie Liu, he received his M.Sc. in Applied Mathematics (2007) from harbin institute of technology. Now he is full lecturer of mathematics at department of mathematics and statistics, Ankang University. His current research interests include different aspects of numerical methods for PDE and mathematical modeling. 